\title{
A novel dispersin protein in enteroaggregative Escherichia coli
}

\author{
Jalaluddin Sheikh, ${ }^{1}$ John R. Czeczulin, ${ }^{1}$ Susan Harrington, ${ }^{1}$ Susan Hicks, ${ }^{2}$ \\ Ian R. Henderson, ${ }^{1}$ Chantal Le Bouguénec, ${ }^{3}$ Pierre Gounon, ${ }^{4}$ Alan Phillips, ${ }^{2}$ \\ and James P. Nataro ${ }^{1,5,6}$ \\ ${ }^{1}$ Center for Vaccine Development, Department of Pediatrics, University of Maryland School of Medicine, \\ Baltimore, Maryland, USA \\ ${ }^{2}$ Royal Free Hospital, London, United Kingdom \\ ${ }^{3}$ Pathogenie Bacterienne des Muqueuses, Institut Pasteur, Paris, France \\ ${ }^{4}$ Institut National de la Santé et de la Recherche Médicale U452, UFR Médecine, Nice, France \\ ${ }^{5}$ Department of Medicine, and \\ ${ }^{6}$ Department of Microbiology and Immunology, University of Maryland School of Medicine, Baltimore, Maryland, USA
}

Enteroaggregative Escherichia coli (EAEC) is a diarrheal pathogen defined by its characteristic aggregative adherence (AA) to HEp-2 cells in culture. We have previously shown that EAEC strains secrete a $10-\mathrm{kDa}$ protein that is immunogenic in a human EAEC challenge model. We report here that this protein is encoded by a gene (called aap) lying immediately upstream of that encoding the AggR transcriptional activator, and that aap is under AggR control. The product of aap has a typical signal sequence and is secreted to the extracellular milieu, where it remains noncovalently attached to the surface of the bacterium. EAEC aap mutants aggregate more intensely than the wild-type parent in a number of assays, forming larger aggregates and fewer individual bacteria. Infection of colonic biopsies with wild-type EAEC strain 042 and its aap mutant revealed more dramatic autoagglutination of the mutant compared with the wild-type parent. Our data suggest that the aap gene product participates in formation of a surface coat that acts to disperse the bacteria, thus partially counteracting aggregation mediated by aggregative adherence fimbriae. We have therefore named the aap gene product "dispersin," and we propose that it may be representative of a functional class of colonization factors. Since dispersin is expressed in vivo, is highly immunogenic, and is present in most EAEC strains, it holds considerable promise as an EAEC immunogen.

J. Clin. Invest. 110:1329-1337 (2002). doi:10.1172/JCI200216172.

\section{Introduction}

Enteroaggregative Escherichia coli (EAEC) is an emerging diarrheal pathogen that has been associated with endemic and epidemic diarrheal illness both in developing and industrialized countries $(1,2)$. The pathogenesis of EAEC infection is thought to comprise adherence to the intestinal mucosa, most likely of both the small and the large intestines, followed by elaboration of one or more enterotoxins (3). These toxins include the heat stable toxin-like EAST1 and plasmid-encoded (Pet) toxin, the latter of which is responsible for mucosal cytotoxicity in explanted colonic

Received for publication June 12, 2002, and accepted in revised form August 27, 2002.

Address correspondence to: James P. Nataro, 685 West Baltimore Street, Baltimore, Maryland 21201, USA. Phone: (410) 706-5328; Fax: (410) 706-6205;

E-mail: jnataro@medicine.umaryland.edu.

Ian R. Henderson's present address is: Division of Immunity and Infection, University of Birmingham Medical School, Edgbaston, Birmingham, United Kingdom.

Conflict of interest: No conflict of interest has been declared. Nonstandard abbreviations used: enteroaggregative Escherichia coli (EAEC); aggregative adherence (AA); aggregative adherence fimbriae (AAF); trichloroacetic acid (TCA). tissue (1, 2, 4-6). Adherence of EAEC to the intestinal mucosa is characterized by the presence of a thick biofilm $(2,7)$, which may mediate persistence of this organism in the human intestine.

The defining feature of EAEC remains its distinctive aggregative adherence (AA) pattern observed in the HEp-2 adherence assay, in which the bacteria are coincubated with HEp-2 cells in cell culture medium for 3 hours. In this assay, EAEC adhere to the surface of HEp-2 cells, to the glass substratum, and to each other in a characteristic stacked-brick formation (8). We have shown that AA to HEp-2 cells in well-characterized EAEC strains requires a member of the aggregative adherence fimbriae (AAF) family $(9,10)$. AAF/I and $\mathrm{AAF} / \mathrm{II}$ are each encoded on an approximately $100-\mathrm{kb}$ virulence plasmid (designated pAA) $(11,12)$. The proven human pathogenic strain 042 requires $\mathrm{AAF} / \mathrm{II}$ fimbriae for adherence to colonic explants (10).

Despite the importance of AAF fimbriae in adherence, we have observed that the majority of EAEC strains lack AAF/I and AAF/II (6). However, our data also suggest that most EAEC strains in our collection carry the pAA plasmid, as evidenced by the presence of several other conserved plasmid loci. Most prominent among these is a transcriptional activator of the $\mathrm{AraC}$ 
class, designated $\mathrm{AggR}$, which is required for expression of both AAF/I and AAF/II, but which is also present in a large percentage of EAEC strains that do not express any identified AAF. A second, cryptic locus (originally designated aspU) was found in $80 \%$ of EAEC strains in our collection (6). Here, we characterize the as $p U$ locus, which lies immediately upstream of aggR in EAEC 042. This gene encodes a secreted low-molecular weight protein that appears to coat the bacterial surface and thereby promote dispersal of EAEC on the intestinal mucosa. Importantly, the protein, designated dispersin herein, corresponds to a previously cryptic protein against which EAEC-challenged volunteers demonstrated secretory IgA responses (9).

\section{Methods}

Cloning and molecular biology methods. Standard molecular biology techniques used published methods (13). E. coli DH5 $\alpha$ was used as the host strain for recombinant DNA experiments unless otherwise noted (Table 1).

A mutation in the aap gene was constructed using single-crossover insertion mutagenesis as previously described (12). A DNA fragment internal to the aap gene was synthesized by PCR, and the fragment was cloned into the KpnI and SacI sites of suicide vector PJP5603 (14). Primers used to amplify the internal fragment had the following sequence, with restriction sites underlined: 5'-ATGGTACCTTGTTATCTTTTCTGGCATCTTGGGT; 5'-ATGAGCTCGAGGGGGTAACAACCCCTTTGAAGT.

A previously constructed aggR mutant strain, JS28, was complemented for AggR expression under control of the ara promoter in pBAD30. The aggR gene was amplified from 042 by PCR by using the following primers: AggR-forward primer, 5'-GCCCCGGGATGAAATTAAAACAAAACATCGAAAA-3'; and AggR-reverse primer, 5'-GCCCCGGGCTATTGGCTTTTAAAATAAGTCAAG-3'. The product was digested with SmaI and cloned into the SmaI site of expression vector PBAD30. $P C R$ and RT-PCR for aap-aggR. PCR was performed to assess the conservation of the aap-aggR locus using the following primers: Aap-forward, 5'-ATGAAAAAAATTAAGTTTGTTATCTT-3'; and AggR-reverse, 5'-GCCCCGGGCTATTGGCTTTTAAAATAAGTCAAG-3'.

RT-PCR reactions were performed from Luria broth cultures as previously described (15). Primers used for cDNA synthesis were cat (5'-CCACTCATCGCAGTACTGTT), aap (5'-TTATTTAACCCATTCGGTTAGAGC), and aggR (5'-GCCCCGGGCTATTGGCTTTTAAAATAAGTCAAG). For the PCR step, the reverse primers were as above, paired with the following forward primers: cat (5'-TCACTGGATATACCACCGTT), aap (5'-ATGAAAAAAATTAAGTTTGTTATCTT), and aggR (5'-GCCCCGGGATGAAATTAAAACAAAACATCGAAAA).

Expression and purification of the aap gene product. To facilitate purification of the aap gene product, the aap gene was ligated into the SphI-BamHI sites of plasmid PQE70 downstream of the lac promoter. The resulting plasmid, pAap, expresses dispersin fused to six histidine residues at the C-terminus. The aap gene was amplified by PCR using primers with the following sequences: $5^{\prime}$-ACATGCATGCAAAAAATTAAGTTTGTTATC, and 5'-CGGGATCCAACCCATTCGGTTAGAGC. Amplification results in a DNA fragment with SphI and BamHI restriction sites at the aap upstream and downstream ends, respectively (underlined nucleotides in primers above). The PCR product was digested with SphI and BamHI and ligated into similarly digested vector $\mathrm{PQE70.}$

The Aap-6His fusion protein was purified from a 100 -ml culture volume grown in L-broth with shaking at $37^{\circ} \mathrm{C}$ to an $\mathrm{OD}_{600}$ of 0.6. At this point, IPTG (Sigma Chemical Co., St. Louis, Missouri, USA) was added to a final concentration of $1 \mathrm{mM}$, and incubation at $37^{\circ} \mathrm{C}$ was continued for an additional 4 hours. Extraction of the protein was performed according to manufacturer's protocol no. 10 (QIAexpress manual version 3/99; QIAGEN Inc., Valencia, California, USA).

Polyclonal antiserum was raised against the purified Aap-6His protein fusion by subcutaneous injection of rabbits with Aap protein excised from SDS-PAGE gels as described (16). We performed dot blots for AAF/II expression by spotting $100-\mu \mathrm{l}$ aliquots from shaking L-broth cultures onto nitrocellulose paper. Blots were then processed using a 1:500 dilution of anti-AAF/II antiserum previously described, and then developed by standard methods using goat anti-rabbit IgG as previously reported (9).

Localization of the aap gene product. Overnight L-broth cultures were diluted 1:20 in $5 \mathrm{ml}$ fresh L-broth and grown to $\log$ phase. The cells were pelleted for $15 \mathrm{~min}$ utes at $1,100 \mathrm{~g}$, and both supernatant and pellet fractions were recovered. The supernatant was precipitated by addition of trichloroacetic acid (TCA) to final concentration of $20 \%$. TCA precipitate was collected by centrifugation at $14,000 \mathrm{~g}$. Loosely adherent surface protein was removed by (a) addition of Triton X-100 detergent (Sigma Chemical Co.) to the L-broth culture (final concentration $0.1 \%$ ) 1 hour before harvesting of cells; (b) heating of the culture to $60^{\circ} \mathrm{C}$ for 20 minutes prior to separation of whole cells and supernatant; or (c) vortexing of a 5-ml culture in a VWR Vortex Genie (VWR Scientific, Bridgeport, New Jersey, USA) at the highest setting for 10 minutes.

Adherence and autoagglutination assays. The HEp-2 adherence assay was performed as previously described (17). In certain experiments, purified Aap or BSA (Sigma Chemical Co.) was added to the cells in fresh medium immediately prior to the addition of the bacterial inoculum. Glass-adherence assays were performed as described (15). The autoagglutination assay was performed as described by Hasman et al. (18). Quantitation of adherent organisms was performed by triplicate plate count of serial dilutions removed by 10 minutes' incubation in $1 \%$ Triton X-100.

Mucus penetration assay. Mucus penetration was measured by a modification of the method described by Lehker and Sweeney (19). Cells grown overnight in L-broth were diluted 1:200 in L-broth and grown to mid-log phase for 3 hours. Bacteria were pelleted by 


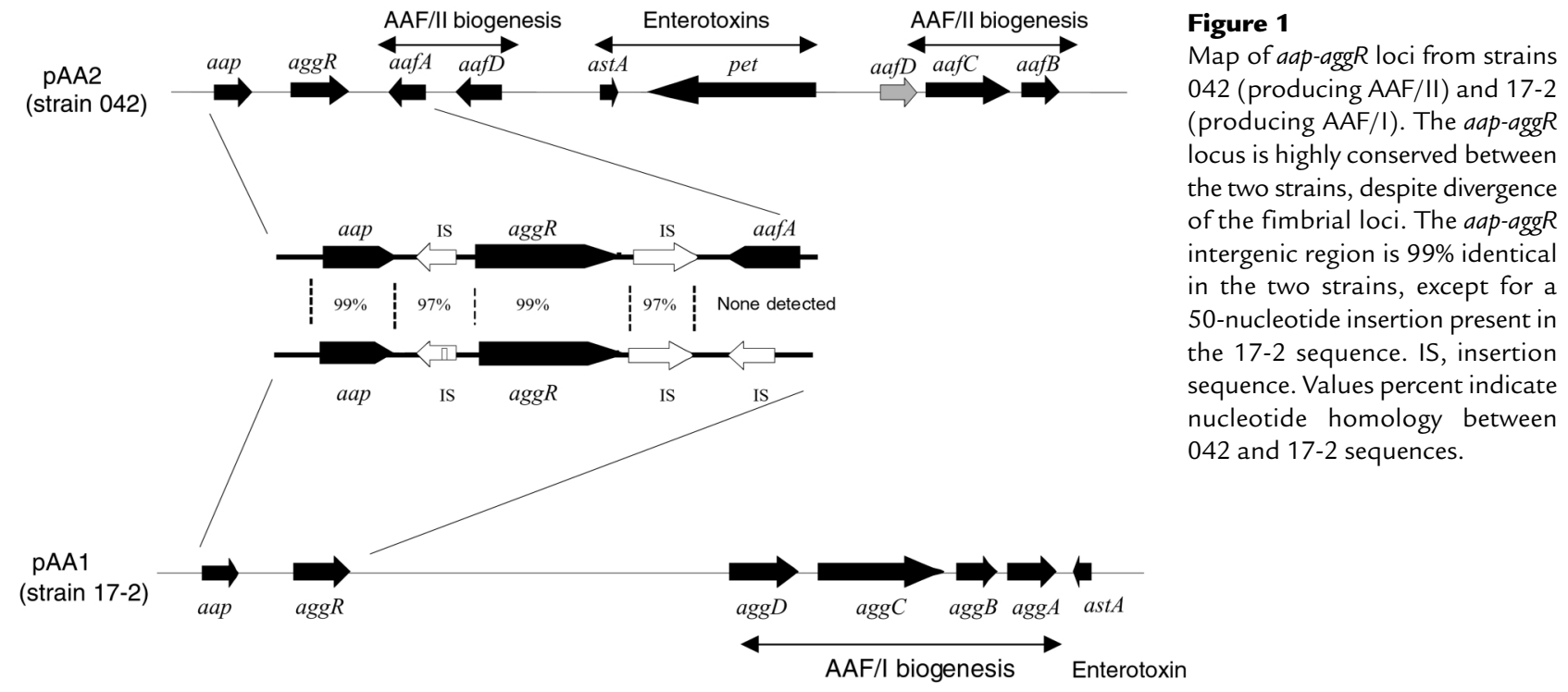

centrifugation and resuspended in PBS ( $\mathrm{pH} 7.4)$ to $1 \times 10^{7}$ cells $/ \mathrm{ml}$. Bovine submaxillary mucin (Sigma Chemical Co.) was prepared to a concentration of 10 $\mathrm{mg} / \mathrm{ml}$ in HBSS (GIBCO BRL; Life Technologies Inc, Gaithersburg, Maryland, USA.) and was loaded into a $1-\mathrm{ml}$ tuberculin syringe fitted with a stopcock and 22 -gauge needle, creating a mucous column. Ten microliters of cell suspension was gently layered onto the mucin, using a separate column for each strain. The column was incubated at $37^{\circ} \mathrm{C}$ for 75 minutes in an upright position. Fractions consisting of ten drops each were collected from the bottom of the column. The starting inoculum and each fraction were plated to L agar media with appropriate antibiotics to determine the number of CFUs per $\mathrm{ml}$.

In vitro organ culture. The effect of the aap mutation was studied on colonic tissue using pediatric intestinal biopsies in an in vitro organ culture model as previously described (20). Histologically normal samples of transverse colon from six pediatric patients (five male, one female; aged 41-174 months; median age 107 months) were obtained during routine endoscopic examination after ethical approval and informed parental consent.

Electron microscopy. Immunogold transmission electron microscopy for detection of Aap was performed on overnight Luria broth cultures as previously described $(10,21)$. For scanning electron microscopy, strains were grown in L-broth overnight with shaking at $37^{\circ} \mathrm{C}$. Thirty microliters of an overnight bacterial culture was spotted onto silica chips (Ted Pella Inc., Redding, California, USA) previously coated with $0.1 \%$ polylysine (Sigma Chemical Co.) and allowed to incubate at room temperature for 5 minutes. The suspension was withdrawn using a Pasteur pipette; then $2 \%$ glutaraldehyde (Electron Microscopy Sciences Inc., Fort Washington, Pennsylvania, USA) in CaCo buffer ( $0.1 \mathrm{M}$ Ca cacodylate, 3 $\mathrm{mM} \mathrm{CaC} 1_{2}$; all reagents from Electron Microscopy Sciences Inc.) was applied for 1 hour and incubated at room temperature. Liquid was again withdrawn, and $0.1 \mathrm{M}$ CaCo buffer was applied three times for 5 minutes per wash. The specimen was then post-fixed with $2 \%$ osmium tetroxide (Electron Microscopy Sciences Inc.) in $\mathrm{CaCo}$ buffer for 1 hour on ice. The specimens were rinsed twice with distilled water for 5 minutes each. Specimens were then stained with $2 \%$ uranyl acetate (Ted Pella Inc.) for 30 minutes at room temperature, rinsed with $50 \%$ ethanol, then dehydrated for 5 minutes each in sequential baths of $50 \%, 70 \%, 90 \%$, and $100 \%$ ethanol. The $100 \%$ ethanol wash step was performed three times for 5 minutes each. The specimens were then inserted into a critical point dryer (model CPD-030; BAL-TEC AG, Balzers, Switzerland) until dry, followed by sputter coating in a Desk II Cold Sputtercoater for 90 seconds using platinum and paladium according to the manufacturer's protocols (Denton Vacuum Inc., Moorestown, New Jersey, USA). Specimens were then examined by scanning electron microscopy in a LEO 1550 field emission scanning electron microscope (LEO Electron Microscopy Inc., Thornwood, New York, USA).

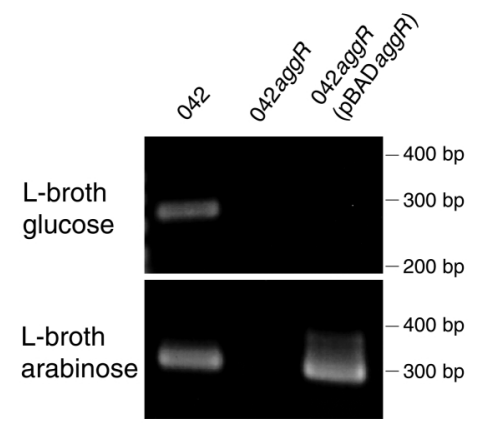

Figure 2

RT-PCR for aap transcript in 042, 042aggR, and 042aggR(pBADaggR). Cultures were grown to late log phase in either L-broth with $0.5 \%$ glucose or L-broth with $0.5 \%$ arabinose. RT-PCR was performed as described in Methods, and products were separated on $1 \%$ agarose gels. The aap transcript migrates as a $0.3-\mathrm{kb}$ species as predicted. 


No
treatment

\section{Figure 3}

Localization of the aap gene product. Western immunoblot analysis was performed on 042 grown in L-broth and subjected to addition of $0.1 \%$ Triton $\mathrm{X}-100$ detergent, incubation at $60^{\circ} \mathrm{C}$ for 20 minutes, or vigorous vortexing for 10 minutes. Cultures were grown to late log phase, and bacteria were pelleted by centrifugation. Pellets $(P)$ were prepared by boiling whole pellet in SDS-PAGE buffer and separating $10 \%$ of the sample derived from a $1.0-\mathrm{ml}$ culture. Supernatant (S) was prepared by TCA precipitation. Western immunoblots were performed by standard methods using highly specific polyclonal antiserum to Aap protein. Aap migrates at the predicted 10-kDa size.

\section{Results \\ Characterization of the aap locus. We have previously report- ed that aggR, encoding a transcriptional activator of AAF expression, is encoded downstream of the fimbrial sub- unit (aafA) on PAA2 in prototype EAEC strain 042 (Fig- ure 1). Sequencing of plasmid DNA upstream of aggR revealed an open reading frame (ORF) terminating 815 nucleotides from the aggR start codon. The ORF was 348 nucleotides in length, encoding a predicted protein product of 116 amino acids. SignalP analysis strongly predicted a signal sequence with cleavage after position 21 . The predicted $\mathrm{N}$-terminal amino acid sequence of the mature species was identical to the $\mathrm{N}$-terminus of a cryp- tic protein we had previously reported to be secreted from EAEC strain 17-2, and initially surmised to be a fimbrial subunit (ref. 9; GenBank accession no. A49221). The predicted mature protein product of aap is $10.2 \mathrm{kDa}$ in size. Neither the nucleotide sequence of aap nor its deduced amino acid sequence displays significant iden- tity to any other protein or gene in the databases.}

The aap-aggR region was remarkably conserved between AAF/I-encoding strain 17-2 (GenBank accession no. Z32523) and AAF/II-encoding strain 042 (Figure 1), despite the fact that the organizations of AAF/I and $\mathrm{AAF} / \mathrm{II}$ biogenesis genes are dramatically different $(12,22)$. To assess the conservation of the aap-aggR locus among EAEC strains, PCR was performed on total genomic DNA from a selection of EAEC strains previously hybridized with an aggR probe (6). Primers were chosen from aggR and aap in strain 042 such that the PCR product would span the intergenic region. All strains known to hybridize with the aggR probe yielded identical 2-kb fragments; all probe-negative strains did not yield a PCR product (not shown).

Since aap and $\operatorname{agg} R$ are separated by less than $1 \mathrm{~kb}$, we asked whether or not the two genes were transcriptionally linked. RT-PCR was performed using the aap upstream primer and the aggR downstream primer from the experiments above. This RT-PCR reaction did not yield a product, suggesting that the two are not transcribed polycistronically (not shown).

aap transcription is AggR-dependent. AggR is required for transcriptional activation of $\mathrm{AAF} / \mathrm{I}$ and $\mathrm{AAF} / \mathrm{II}$ genes, and its proximity to aap suggested that AggR might also be required for aap expression. To test this hypothesis, we performed RT-PCR for the aap transcript in strains 042 and $042 \operatorname{agg} R$ (Figure 2). A product of the predicted size was seen in 042 but was absent in $042 a g g R$. To confirm a requirement for AggR, we complemented $042 \operatorname{agg} R$ with $\operatorname{agg} R$ cloned under control of the arabinose-dependent promoter in pBAD30. Restoration of aap expression was observed in 042(pBADaggR) when cells were grown in the presence of arabinose (ara-inducing conditions) but not when they were grown in the presence of glucose (repressing conditions).

Localization of the aap gene product. The aap gene product has a strongly predicted signal sequence, and previous

\footnotetext{
Figure 4

Electron microscopy of 042 and mutants. (a) Immunogold transmission electron microscopy for Aap protein. Black arrows indicate surface protein detected by antiAap antibody. Strains examined by scanning electron microscopy are 042 (b and c), 042aafA (d), 042aap $(\mathbf{e}-\mathbf{g})$, and 042aap(pAap) (h). In i, 042aap was incubated with 3 $\mathrm{mg} / \mathrm{ml}$ purified Aap protein prior to fixing and staining. White arrows indicate AAF fimbriae in all specimens. All bars indicate $1 \mu \mathrm{m}$.
}
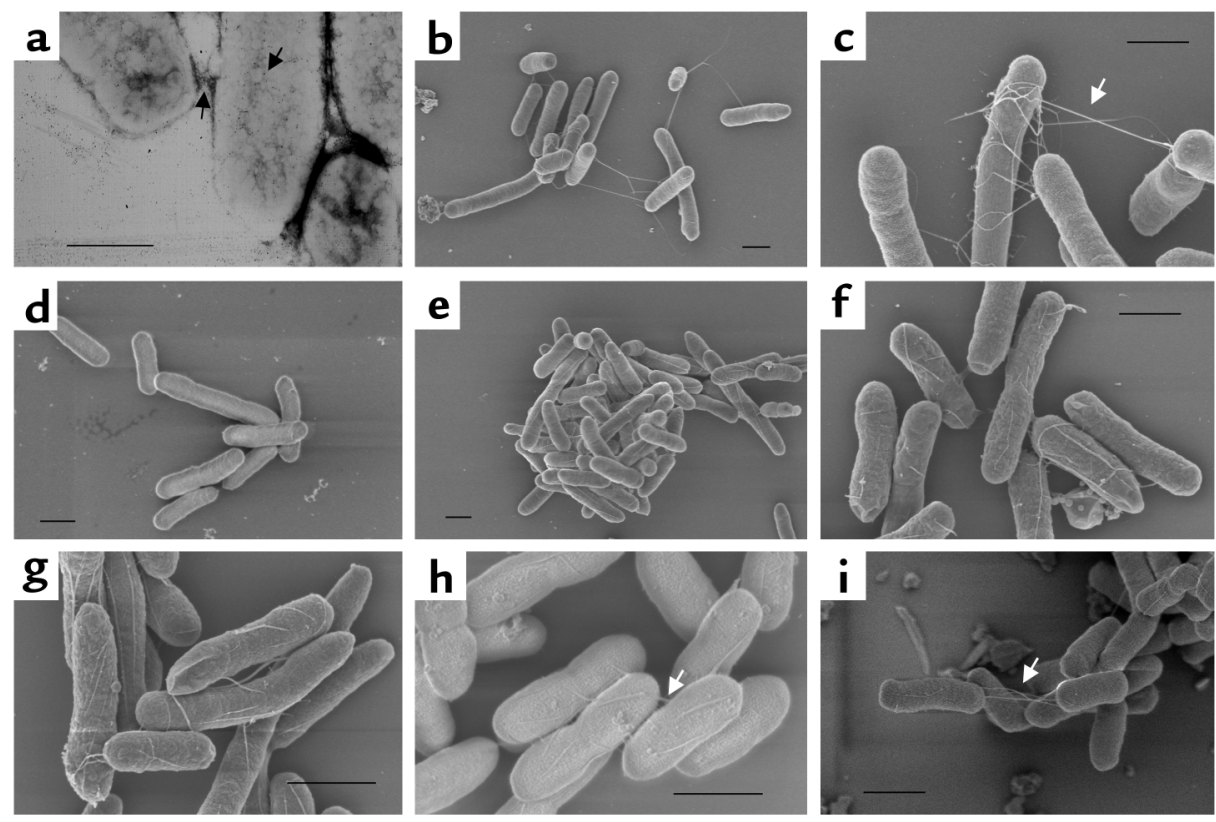
a
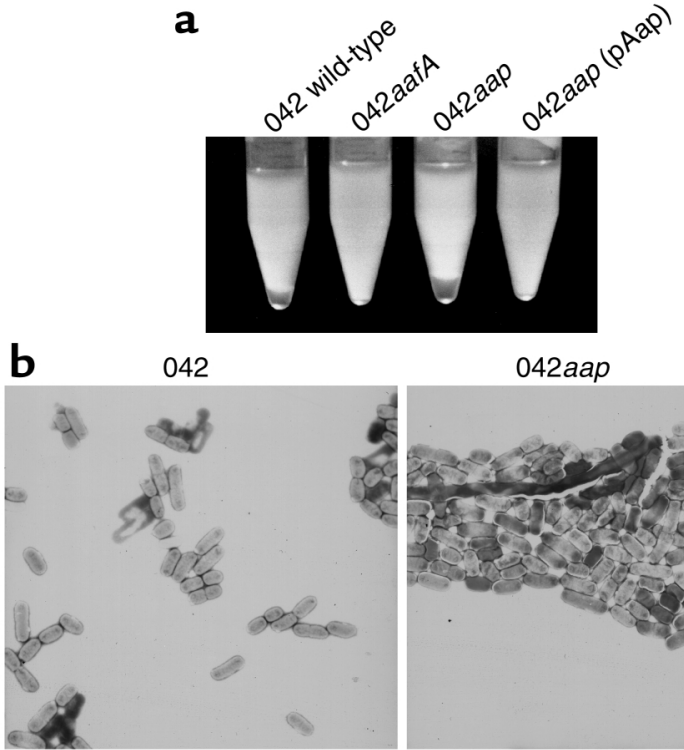

042aap

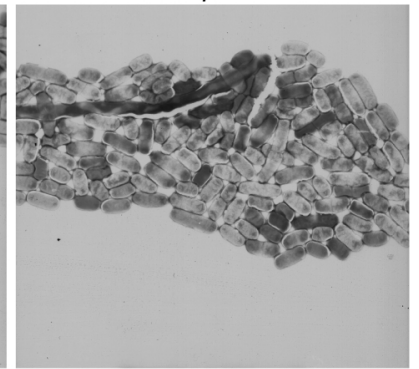

Figure 5

Autoagglutination of 042 and mutants in L-broth. L-broth cultures were grown overnight at $37^{\circ} \mathrm{C}$ with shaking, then permitted to settle undisturbed at room temperature for 1 hour. (a) Broth cultures revealing a pellet at the bottom of the culture tube correlating with autoagglutination of bacterial cells. (b) Transmission electron microscopy of 042 and 042 aap cultures. Ten microliters of L-broth cultures from a were withdrawn prior to incubation on the bench-top and processed for negative staining. Bars, $1 \mu \mathrm{m}$.

data have shown that the protein can be found in bacterial supernatants (9). However, quantitative immunoblot experiments suggested that most of the Aap protein pelleted with the bacterium. Experiments were performed to resolve this discrepancy (Figure 3). Broth cultures of 042 were heated to $60^{\circ} \mathrm{C}$ for 20 minutes or subjected to vigorous vortexing. Both heat and shear forces remove bacterial fimbriae (23). Heat treatment of the culture slightly increased the amount of protein found in the supernatant; vortexing did not release Aap. In contrast, addition of Triton X-100 to a final concentration of $0.1 \%$ released the majority of Aap into the supernatant fraction. These data suggest that the aap gene product is a peripheral membrane protein, which is noncovalently attached to the bacterial outer membrane.

Electron microscopy of aap mutants. The aap gene was inactivated in strain 042 by single-crossover homologous recombination of suicide plasmid pJP5603. This construct was complemented by cloning aap into plasmid PQE70, under control of the lac promoter, and was designated pAap. By Immunogold electron microscopy, the surface layer formed a reticulated structure (Figure 4a), which occasionally was visualized between adjacent bacteria (arrows in Figure 4a). 042aap did not stain with the antiserum.

Scanning electron microscopy was also performed on 042 and several mutants (Figure 4, b-i). Under the growth conditions of the assay, 042 expresses only the $\mathrm{AAF} / \mathrm{II}$ fimbriae, as null mutants in the fimbrial subunit are afimbriate (Figure $4 \mathrm{~d}$ ). AAF/II extends from the bacterial cell as thin fibers (Figure 4, b and c), joining into thicker bundles and often separating again before attaching the bacterium to the substratum. AAF/II-mediated interbacterial adherence commonly occurred at distances of $2 \mu \mathrm{m}$ or more (Figure $4 \mathrm{c}$ ). This appearance was consistent with previous reports (10).

In contrast, 042aap agglutinated more intimately than its wild-type parent, with attachment at a distance seen very rarely (Figure $4, \mathrm{e}-\mathrm{g}$ ). Interbacterial adherence typically featured bacteria in parallel, with membrane fusions between two bacteria commonly observed (Figure 4f). Moreover, the AAF fimbriae were not observed to extend from the surface of the bacteria but rather lay along the bacterial cell surface, often wrapping around the bacterium (Figure 4, fand g). The complemented aap mutant exhibited an intermediate phenotype (Figure $4 \mathrm{~h}$ ), with looser aggregation than that of 042aap. The addition of purified Aap protein to 042aap resulted in partial rescue of the wild-type phenotype (Figure 4i).

Altered adherence and autoagglutination in an aap mutant. Because of its location near adherence-related genes in EAEC strains and the appearance of 042aap under scanning electron microscopy, we suspected that the aap gene product may play a role in EAEC autoagglutination and adherence. Indeed, stationary-phase broth culture of 042aap exhibited differences in spontaneous settling of bacterial aggregates to the bottom of the culture tube (Figure 5). This difference was reversed in 042aap(pAap) (Figure 5a). Increased aggregation of 042aap was confirmed by transmission electron microscopy (Figure 5b). Aggregation of bacteria in broth cultures was quantitated using the settling assay described by Hasman et al. (18). A typical result of this assay is depicted in Figure 6. In the conventional 3-hour HEp-2 adherence assay, 042aap displayed dramatically larger aggregates than did its wild-type parent (Figure 7, a and b). This

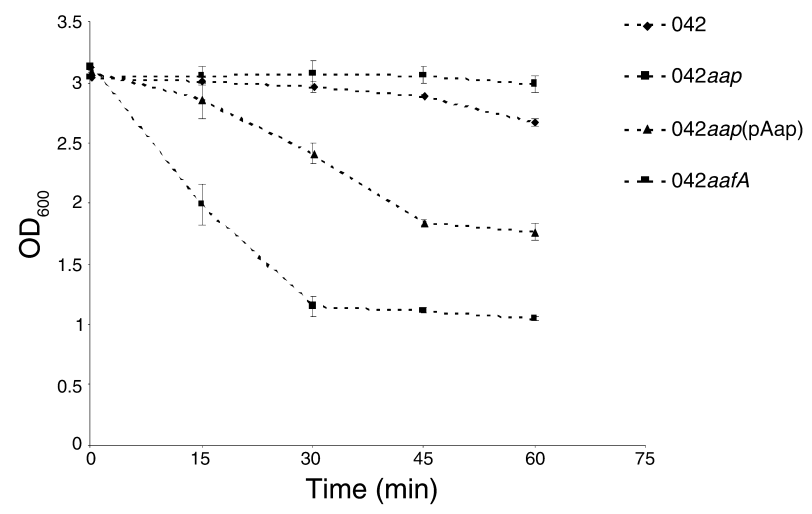

\section{Figure 6}

Spontaneous settling of 042 and mutants. Overnight L-broth cultures containing $0.2 \mathrm{mM}$ IPTG were permitted to settle undisturbed at room temperature. At 15-minute intervals, a $100-\mu \mathrm{l}$ sample was withdrawn from the top of each broth culture and the $\mathrm{OD}_{600}$ of the withdrawn sample was determined. Rate of clearing of the uppermost portion of the culture correlates with rate of spontaneous settling, a measure of agglutination. Samples were tested in triplicate. Bars represent SEM. 

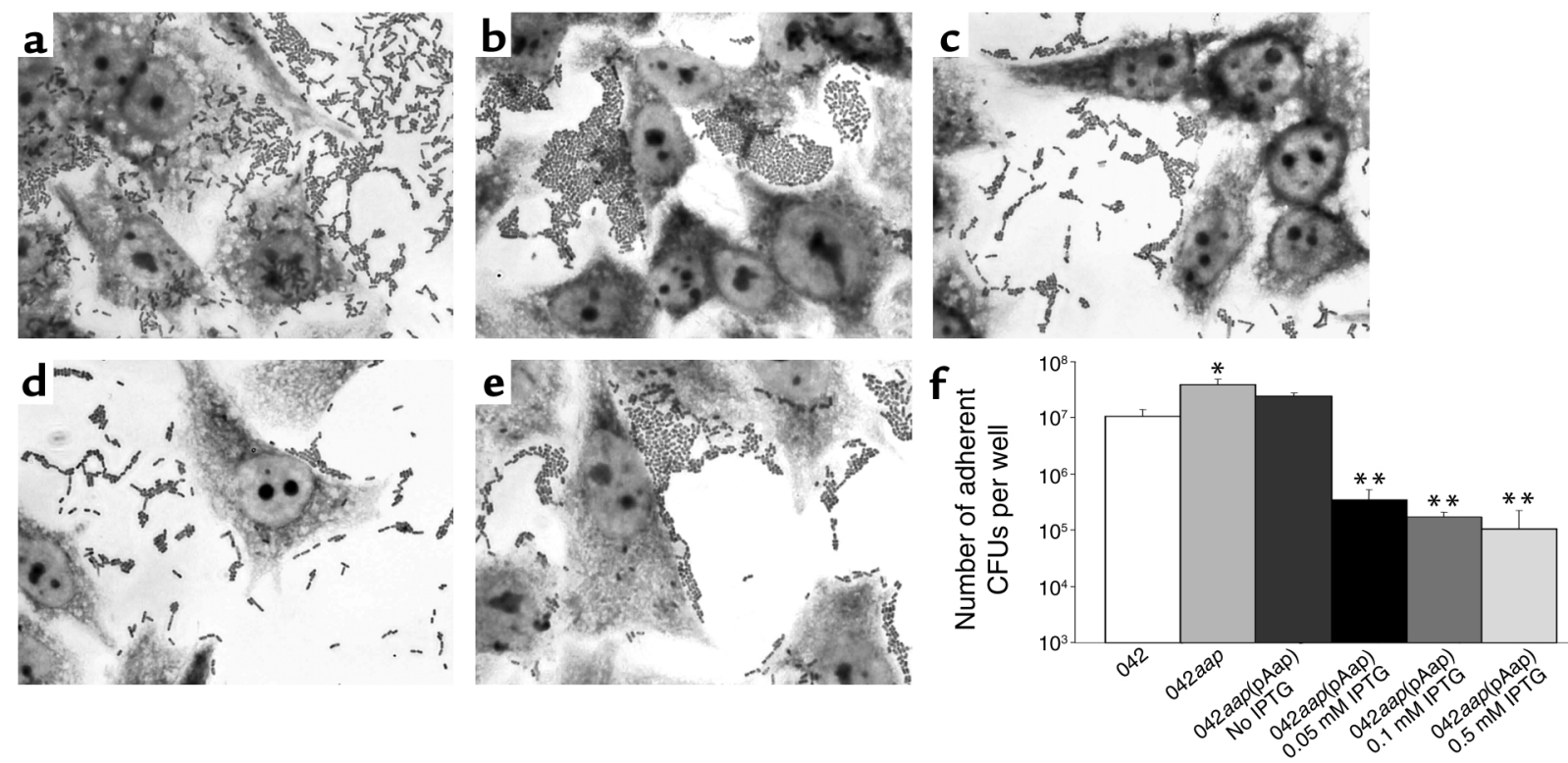

Figure 7

Role of aap in epithelial cell adherence. Assays were performed for 3 hours in DMEM-0.5\% glucose with $0.2 \mathrm{mM} \mathrm{IPTG} \mathrm{at} 37^{\circ} \mathrm{C}$ in atmosphere. (a) 042; (b) 042aap; (c) 042aap(pAap). (d and e) The standard HEp-2 cell adherence assay was performed using strain 042aap, but with pure Aap (d) or pure BSA (e) added concurrently with the bacterial inoculum. Each protein was added to a final concentration of 0.1 $\mathrm{mg} / \mathrm{ml}$. (f) Quantitation of HEp-2 cell adherence on confluent monolayers growing in DMEM without IPTG (042 and 042 aap) or with indicated IPTG concentration [042aap(pAap)]. Bacteria were removed from glass using 1\% Triton X-100 and quantitated by viable count. Assays were performed in triplicate, and means are illustrated in histograms with error bars indicating SE. ${ }^{*} P<0.05$ vs. 042 by one-tailed $t$ test of log-transformed data; ${ }^{*} P<0.05$ vs. $042 a a p$ by one-tailed $t$ test of log-transformed data.

phenotype was also reversed in 042aap(pAap) (Figure 7c). When Aap protein was added to the HEp-2 adherence assay simultaneously with bacterial inoculation, adherence by 042 aap returned to levels similar to those seen with wild-type (Figure 7d). Addition of BSA (Figure $7 \mathrm{e}$ ) with the inoculum or addition of Aap after inoculation (not shown) did not have any effect on adherence. Quantitation of bacterial adherence by plate count revealed a dose-response relationship to IPTG concentration in 042aap(pAap), with two-log reduction in adherence upon addition of $0.05 \mathrm{mM}$ IPTG. This concentration of IPTG does not affect growth rate of the test bacteria (not shown).
When a similar adherence assay was performed in the absence of HEp-2 cells (Figure 8), 042aap produced more abundant adherence to glass than did its parent, whereas adherence of 042aap(pAap) was similar to that of wild-type. Quantitation of this assay (Figure 8d) also revealed a dose-response relationship to IPTG concentration.

Roles for aap in mucosal adherence and mucous layer penetration. We have previously shown that EAEC strain 042 adheres abundantly to the surface of human intestinal biopsy sections in culture $(24,25)$. To investigate the role of the aap gene product in mucosal adherence, 042, 042aap, and 042aap(pAap) were incubated with
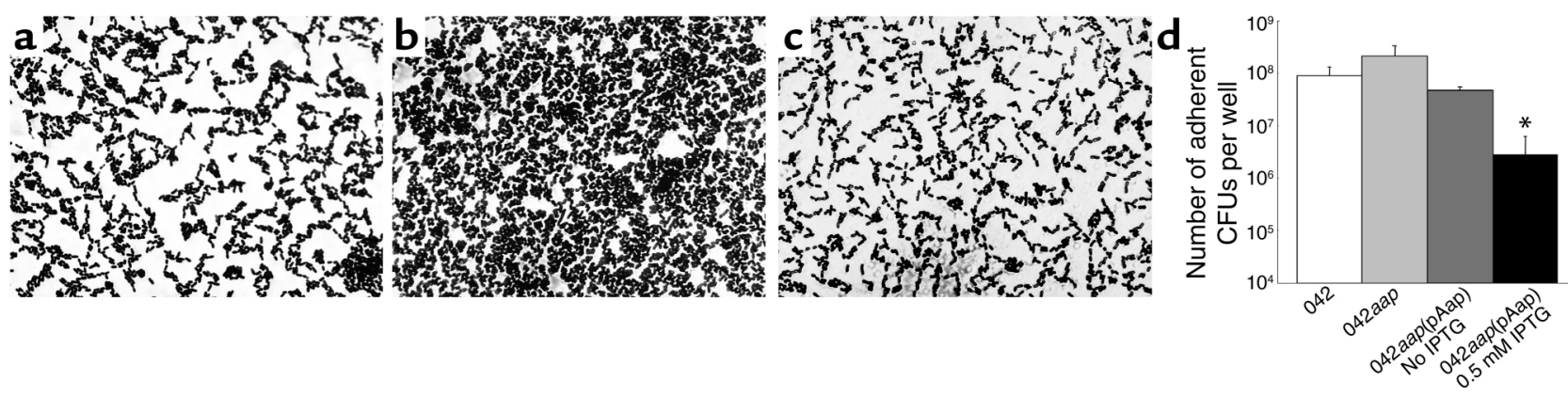

\section{Figure 8}

Glass adherence assay. (a-c) Assay was performed for 3 hours in DMEM-0.5\% glucose with $0.1 \mathrm{mM} \mathrm{IPTG}$ at $37^{\circ} \mathrm{C}$ in atmosphere. (a) 042; (b) 042aap; (c) 042aap(pAap). (d) Quantitation of adherence grown in DMEM without IPTG (042 and 042aap) or with the indicated concentration of IPTG [(042aap(pAap)]. Bacteria were removed from glass using $1 \%$ Triton X-100 and quantitated by viable count. Assays were performed in triplicate, and means are illustrated in histograms with error bars indicating SEM. ${ }^{*} P<0.05$ vs. 042 aap by onetailed $t$ test of log-transformed data. 

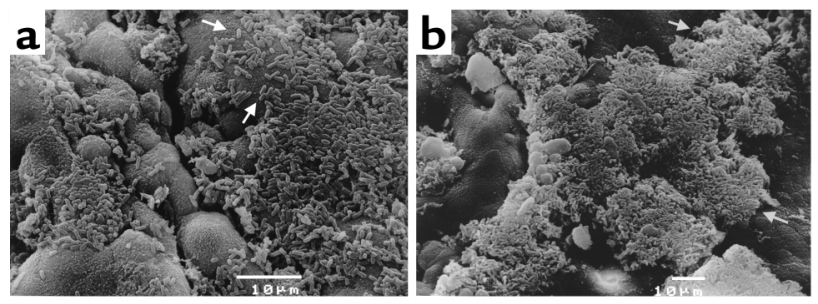

Figure 9

In vitro organ culture assay of 042 (a) and 042aap (b). Bacterial cultures were incubated with colonic biopsies from normal children for 6 hours; then specimens were fixed, stained, and examined by scanning electron microscopy. White arrows (a) denote loose adherence of 042; gray arrows (b) show tight aggregates typical of 042aap. Bars, $10 \mu \mathrm{m}$.

fresh colonic biopsy specimens for 6 hours (Figure 9). Wild-type 042 adhered as previously described, producing a biofilm on the absorptive surface of the colonic mucosa, while sparing the colonic glands. Notably, 042 exhibited both aggregates and individual bacteria, very similar to the scanning electron microscopic images from silica chip experiments shown in Figure 4. In contrast, 042aap adhered in larger, tighter aggregates with very few single bacteria observed on the surface of the mucosa.

We hypothesized that decreasing the number of AAF-mediated aggregates could serve to facilitate the penetration of the 042 through the intestinal mucous layer, on the assumption that single bacteria would be more adept at swimming through the mucous gel. To test this hypothesis, we adapted a previously published method (19) in which bacteria are placed at the top of a column composed of purified mucin in water. These experiments revealed that 042aap was more than an order of magnitude less efficient at penetrating the mucin column than was the wild-type parent strain (Figure 10).

Secretion of Aap. Unresolved by the above experiments is the mechanism of Aap translocation past the outer membrane. We observed above that the predicted aap gene product possesses several attributes characteristic of bacterial pilin subunits, including a C-terminal glycine residue that mediates interaction with a PapDlike chaperone protein (26). We have previously shown that AAF/II expression is dependent on the PapD-like chaperone AafD and the PapC usher homolog AafC (12). To assess a possible role for the AafC/AafD secretion system in Aap expression, we assayed Aap in supernatants of 042 with insertional mutations in the aafD or aafC genes. Both mutants secreted Aap (Figure 11). Since aafD and aafC mutants are afimbriate (12), this experiment provides further verification that, though secreted, the Aap protein is not incorporated into the AAF organelle.

\section{Discussion}

We have previously shown that most EAEC strains in our collection harbor a partially conserved virulence plasmid (designated PAA), which typically encodes the transcriptional activator AggR. Here, we show that aggR is part of a highly conserved two-gene cluster, which also encodes a novel low-molecular weight secreted protein that mediates dispersal of EAEC on the colonic mucosa. In recognition of this phenotype, we have herein designated this gene aap (anti-aggregation protein). We now further suggest the more descriptive name "dispersin" for the aap gene product. Our data suggest that dispersin is secreted to the environment, but that it remains noncovalently associated with the bacterial cell surface.

Several roles for dispersin in adherence and colonization are plausible. All enteric pathogens must adhere to the epithelial surface. But at the same time, it may be beneficial to negatively modulate adherence to permit dispersal across the epithelial surface. Knutton et al. have shown that the bundle-forming pilus of enteropathogenic E. Coli (EPEC) mediates both aggregation and dispersal, which occur sequentially (27). In this way, EPEC can adhere and multiply early in the pathogenetic sequence, thereby establishing a foothold on the epithelium, but the bacteria then disperse individual progeny, which are free to establish new foci of infection. This paradigm is intuitively beneficial and therefore may be a property of many other mucosal pathogens. Indeed, Benitez et al. have suggested that the Hap mucinase of Vibrio cholerae may serve as an enzymatic "detachase," mutation of which results in increased density of bacterial colonization but also attenuation of virulence (28).

We also hypothesized that dispersin may play a role in penetration of the intestinal mucous blanket, expecting that large aggregates would be retarded in translocation through a viscous gel. Indeed, using a purified mucin gel column assay, we showed that the dispersin mutant penetrated more slowly than the wild-type parent. The human colonic mucosa is covered with a continuous mucous gel approaching $1 \mathrm{~mm}$ in thickness

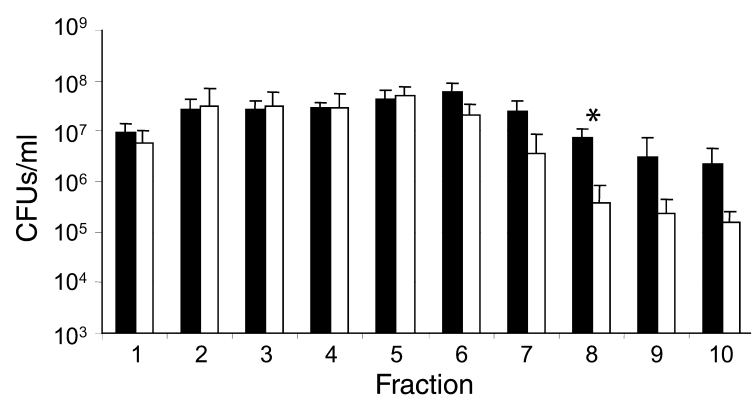

\section{Figure 10}

Penetration of 042 and $042 a a p$ through mucus. Columns of 10 $\mathrm{mg} / \mathrm{ml}$ mucin in HBSS were prepared in tuberculin syringes, and the bacterial inocula were applied to the top of the column. The columns were incubated at $37^{\circ} \mathrm{C}$ for 75 minutes. After this time, the mucin suspension was drained from the column in $0.1-\mathrm{ml}$ fractions, and the number of 042 (black bars) or 042aap (white bars) was determined by quantitative plating in triplicate. Bars represent SD. Fraction 10 represents the bottom of the column, fraction 1 the top. ${ }^{*} P<0.05$ by Student's $t$ test of log-transformed data. 


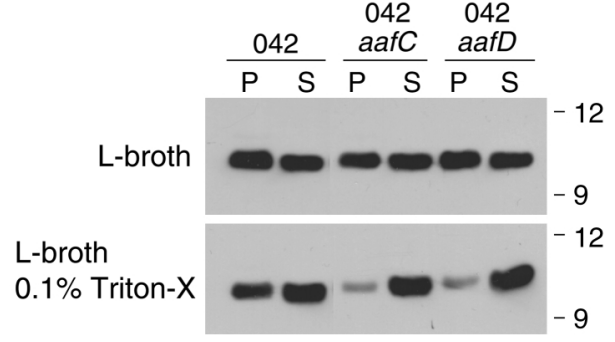

\section{Figure 11}

Western immunoblots for Aap performed on supernatants $(S)$ or pellets $(P)$ of overnight L-broth cultures with or without $0.1 \%$ Triton $\mathrm{X}-100$. Positions of molecular weight markers are shown at right.

(29), which provides a formidable barrier against colonic pathogens. Single bacteria may be more adept at swimming through this layer; alternatively, biophysical characteristics conferred by the dispersin protein coat could promote mucous layer penetration. Whatever their precise roles, Aap, Hap, and some component of bundle-forming pili are apparently the first examples of bacterial "dispersins," which may facilitate efficient colonization via bacterial dispersal. Thorough elucidation of the roles of these dispersins in vivo has not yet been accomplished, because of the limited availability of good whole-animal models, but is clearly worthy of investigation.

We can propose one potential mechanism by which dispersin may promote dispersal. AAF fimbriae are highly hydrophobic (10), and this hydrophobicity would favor strong autoagglutination in an aqueous environment. The predicted amino sequence of dis- persin is more hydrophilic than that of AAF/II, and thus the presence of dispersin on the surface of the bacterial cell may ameliorate surface hydrophobicity. Scanning electron microscopy data are consistent with this hypothesis. Absence of dispersin results in striking alteration in the morphology of the AAF fimbriae. In the absence of dispersin, the fimbriae cling to the surface of the bacterial cell, although their number and diameter do not appear changed. This would imply that the surface of the outer membrane under the dispersin coat is largely hydrophobic; such hydrophobicity could be mediated by outer membrane proteins, or perhaps by other hydrophobic surface structures previously described in EAEC (30).

Notably, despite the fact that aap is coregulated with AAF adhesins and that dispersin has C-terminal characteristics consistent with secretion by the usher-chaperone pathway, we found that mutants in either the usher or the chaperone secreted normal levels of dispersin. Thus, the mechanism by which dispersin is translocated across the outer membrane is as yet unknown. We found it interesting that AAF mutants bound less dispersin protein in the presence of Triton $\mathrm{X}$-100. Immunofluorescence microscopy experiments suggest that some dispersin is bound within AAFmediated aggregates (not shown), providing one possible explanation for this observation.

The requirement for AggR in aap expression is highly significant. AggR has been shown to be required for expression of AAF biogenesis genes (31), yet there are many strains that carry $\operatorname{agg} R$ and do not have an identified AAF adhesin (6). Our data thus suggest that AggR acts as a more versatile regulator of virulence

\section{Table 1}

Strains and plasmids used in this work

\begin{tabular}{|c|c|c|}
\hline Strains & Characteristics & Reference \\
\hline 042 & Wild-type EAEC strain of proven pathogenicity & 32 \\
\hline 042aap & 042 harboring pJP5603 integrated into the aap gene $(\mathrm{Km})$ & This work \\
\hline 042aap(pAap) & 042aap carrying the aap gene cloned into vector PQE70 (Km, Ap) & This work \\
\hline $\mathrm{DH} 5 \alpha\left(\lambda_{\text {pir }}\right)$ & K12 E. coli lysogenized for the pir gene, which permits replication of R6K plasmid replicons & 12 \\
\hline S17-1( $\lambda$ pir $)$ & Conjugative K12 lysogenized for pir & 12 \\
\hline 042aafC & 042 carrying pJP5603 integrated into the gene encoding the AAF/II fimbrial usher gene $(\mathrm{Km})$ & 12 \\
\hline $042 a a f D$ & 042 carrying PJP5603 integrated into the gene encoding the AAF/II fimbrial chaperone gene $(\mathrm{Km})$ & 12 \\
\hline 042aafA & 042 carrying TnphoA inserted into the gene encoding the AAF/II fimbrial subunit gene $(\mathrm{Km})$ & 10 \\
\hline $042 \operatorname{agg} R$ & 042 carrying pJP5603 integrated into the gene encoding the regulator AggR $(\mathrm{Km})$ & 15 \\
\hline $\begin{array}{l}\text { 44-1, 6-1, 17-2, 236, } \\
\text { 199-1, 103-1, 101-1, } \\
\text { 1096, 43996, DS-61R2 }\end{array}$ & Well-characterized, wild-type EAEC strains collected from children in various epidemiologic studies & 6 \\
\hline \multicolumn{3}{|l|}{ Plasmids } \\
\hline PQE70 & Lac-dependent $E$. coli expression vector (Ap) & QIAGEN Inc \\
\hline pJP5603 & R6K suicide plasmid $(\mathrm{Km})$ & 14 \\
\hline pAap & $\begin{array}{l}\text { aap gene cloned into multiple cloning site of PQE70 to provide IPTG-inducible expression } \\
\text { of its gene product as a } 6 \mathrm{His} \text { fusion (Ap) }\end{array}$ & This work \\
\hline pBAD30 & $\begin{array}{l}\text { High-copy number expression vector permitting expression of foreign genes under control } \\
\text { of the arabinose operon promoter }\end{array}$ & 33 \\
\hline pBADaggR & $\begin{array}{l}\text { AggR cloned into multiple cloning site of pBAD } 30 \text { to permit expression of aggR } \\
\text { in the presence of arabinose }\end{array}$ & This work \\
\hline
\end{tabular}

Ap, ampicillin-resistant; $\mathrm{Km}$, kanamycin-resistant. 
genes in EAEC. Indeed, recent data from our laboratory suggest that AggR activates additional unlinked genes on the PAA plasmid and the EAEC chromosome (J. Nishi et al., unpublished results; and E. Dudley et al., unpublished results). Additional characterization of the AggR regulon and its role in EAEC pathogenesis is underway. Coexpression of AAF and dispersin in a common AggR regulon is consistent with an interactive role of the two factors in colonization, but it is interesting that the bacterium would use the same regulator to express both an adhesin and a negative modulator of adhesion.

EAEC is an emerging pathogen, but vaccine development is in its earliest stages. Several obstacles must be overcome before a candidate vaccine can be constructed. Chief among these obstacles is the antigenic heterogeneity of EAEC strains. In addition, given that EAEC infection is a serious health concern predominantly in developing countries, there is little economic incentive toward the development of a vaccine. Therefore, the best approach to EAEC vaccine development would be to identify a highly conserved, highly prevalent, surface-exposed and immunogenic protein that could be expressed in an attenuated vector vaccine. The ideal vector would be one engineered to provide protection against another, perhaps more serious threat (such as shigellosis or typhoid fever). Dispersin fulfills many of these criteria, and experiments are underway to test its feasibility as an EAEC immunogen.

\section{Acknowledgments}

This work was supported by NIH grant AI-33096 to J.P. Nataro. We thank James Kaper for critical review of the manuscript and Alya Raphael for excellent technical assistance.

1. Nataro, J.P., Steiner, T., and Guerrant, R.L. 1998. Enteroaggregative Escherichia coli. Emerg. Infect. Dis. 4:251-261.

2. Nataro, J.P., and Kaper, J.B. 1998. Diarrheagenic Escherichia coli. Clin. Microbiol. Rev. 11:142-201.

3. Okeke, I.N., and Nataro, J.P. 2001. Enteroaggregative Escherichia coli. Lancet Infect. Dis. 1:304-313.

4. Nataro, J.P., Hicks, S., Phillips, A.D., Vial, P.A., and Sears, C.L. 1996. T84 cells in culture as a model for enteroaggregative Escherichia coli pathogenesis. Infect. Immun. 64:4761-4768.

5. Eslava, C.E., et al. 1998. Pet, an autotransporter enterotoxin from enteroaggregative Escherichia coli. Infect. Immun. 66:3155-3163.

6. Czeczulin, J.R., et al. 1999. Phylogenetic analysis of enteroaggregative and diffusely adherent Escherichia coli. Infect. Immun. 67:2692-2699.

7. Tzipori, S., et al. 1992. Studies with enteroaggregative Escherichia coli in the gnotobiotic piglet gastroenteritis model. Infect. Immun. 60:5302-5306.

8. Nataro, J.P., et al. 1987. Patterns of adherence of diarrheagenic Escherichia coli to HEp-2 cells. Pediatr. Infect. Dis. J. 6:829-831.

9. Nataro, J.P., et al. 1992. Aggregative adherence fimbriae I of enteroaggregative Escherichia coli mediate adherence to HEp-2 cells and hemagglutination of human erythrocytes. Infect. Immun. 60:2297-2304.

10. Czeczulin, J.R., et al. 1997. Aggregative adherence fimbria II, a second fimbrial antigen mediating aggregative adherence in enteroaggregative Escherichia coli. Infect. Immun. 65:4135-4145.
11. Savarino, S.J., Fox, P., Yikang, D., and Nataro, J.P. 1994. Identification and characterization of a gene cluster mediating enteroaggregative Escherichia coli aggregative adherence fimbria I biogenesis. J. Bacteriol. 176:4949-4957.

12. Elias, W.P., Jr., Czeczulin, J.R., Henderson, I.R., Trabulsi, L.R., and Nataro, J.P. 1999. Organization of biogenesis genes for aggregative adherence fimbria II defines a virulence gene cluster in enteroaggregative Escherichia coli.J. Bacteriol. 181:1779-1785.

13. Ausubel, F.M., et al. 1999. Current protocols in molecular biology. John Wiley \& Sons. New York, New York, USA. 3.0.1-3.19.8.

14. Penfold, R.J., and Pemberton, J.M. 1992. An improved suicide vector for construction of chromosomal insertion mutations in bacteria. Gene. 118: $145-146$

15. Sheikh, J., Hicks, S., Dall'Agnol, M., Phillips, A.D., and Nataro, J.P. 2001. Roles for Fis and YafK in biofilm formation by enteroaggregative Escherichia coli. Mol. Microbiol. 41:983-997.

16. Harlow, E., and Lane, D. 1999. Using antibodies: a laboratory manual. Cold Spring Harbor Laboratory Press. Cold Spring Harbor, New York, USA. 726 pp.

17. Cravioto, A., Gross, R.J., Scotland, S.M., and Rowe, B. 1979. An adhesive factor found in strains of Escherichia coli belonging to the traditional infantile enteropathogenic serotypes. Curr. Microbiol. 3:95-99.

18. Hasman, H., Chakraborty, T., and Klemm, P. 1999. Antigen-43-mediated autoaggregation of Escherichia coli is blocked by fimbriation. J. Bacteriol. 181:4834-4841.

19. Lehker, M.W., and Sweeney, D. 1999. Trichomonad invasion of the mucous layer requires adhesins, mucinases, and motility. Sex. Transm. Infect. 75:231-238.

20. Hicks, S., Candy, D.C., and Phillips, A.D. 1996. Adhesion of enteroaggregative Escherichia coli to formalin-fixed intestinal and ureteric epithelia from children. J. Med. Microbiol. 44:362-371.

21. Levine, M.M., et al. 1984. Coli surface antigens 1 and 3 of colonization factor antigen II-positive enterotoxigenic Escherichia coli: morphology, purification, and immune responses in humans. Infect. Immun. 44:409-420.

22. Savarino, S.J., Fox, P., Deng, Y., and Nataro, J.P. 1994. Identification and characterization of a gene cluster mediating enteroaggregative Escherichia coli aggregative adherence fimbria I biogenesis. J. Bacteriol. 176:4949-4957.

23. Schoolnik, G. 1994. Purification of somatic pili. In Bacterial pathogenesis. Volume 236. V. Clark and P. Bavoil, editors. Academic Press Inc. San Diego, California, USA. 271-282.

24. Nataro, J., Hicks, S., Phillips, A., Vial, P., and Sears, C. 1996. T84 cells in culture as a model for enteroaggregative Escherichia coli pathogenesis. Infect. Immun. 64:4761-4768.

25. Hicks, S., Candy, D., and Phillips, A. 1996. Adhesion of enteroaggregative Escherichia coli to pediatric intestinal mucosa in vitro. Infect. Immun. 64:4751-4760.

26. Soto, G.E., et al. 1998. Periplasmic chaperone recognition motif of subunits mediates quaternary interactions in the pilus. EMBO J. 17:6155-6167.

27. Knutton, S., et al. 1999. The type IV bundle-forming pilus of enteropathogenic Escherichia coli undergoes dramatic alterations in structure associated with bacterial adherence, aggregation and dispersal. Mol. Microbiol. 33:499-509.

28. Benitez, J.A., et al. 1997. Adherence of Vibrio cholerae to cultured differentiated human intestinal cells: an in vitro colonization model. Infect. Immun. 65:3474-3477.

29. Atuma, C., Strugala, V., Allen, A., and Holm, L. 2001. The adherent gastrointestinal mucus gel layer: thickness and physical state in vivo. Am.J. Physiol. Gastrointest. Liver Physiol. 280:G922-G929.

30. Wai, S.N., Takade, A., and Amako, K. 1996. The hydrophobic surface protein layer of enteroaggregative Escherichia coli strains. FEMS Microbiol. Lett. 135: 17-22.

31. Nataro, J.P., Yikang, D., Yingkang, D., and Walker, K. 1994. AggR, a transcriptional activator of aggregative adherence fimbria I expression in enteroaggregative Escherichia coli.J. Bacteriol. 176:4691-4699.

32. Nataro, J.P., et al. 1995. Heterogeneity of enteroaggregative Escherichia coli virulence demonstrated in volunteers. J. Infect. Dis. 171:465-468.

33. Guzman, L.M., Belin, D., Carson, M.J., and Beckwith, J. 1995. Tight regulation, modulation, and high-level expression by vectors containing the arabinose PBAD promoter. J. Bacteriol. 177:4121-4130. 